\title{
The induction of oral immunotherapy is facilitated by adjunction of omalizumab in children with very severe cow's milk allergy
}

\author{
Anaïs Valeille ${ }^{1}$, François Payot ${ }^{1}$, Sylvie-Anne Andre-Gomez ${ }^{1}$, Florence Villard-Truc ${ }^{1}$, \\ Lorna Garnier $^{2}$, Anne-Karine Corréard ${ }^{1}$, Priscille Biermé ${ }^{1}$, and Camille Braun ${ }^{1}$ \\ ${ }^{1}$ Hopital Femme Mere Enfant \\ ${ }^{2}$ Lyon Sud Hospital
}

June 18, 2020

To the editor:

Cow's milk allergy (CMA) is the most common food allergy in children, with a prevalence of 1 to $3 \%$ (1). While the vast majority of CMAs clear after five years (2), some children have a persistent and severe disease associated with a risk of life-threatening allergic reactions (3). For those patients, oral immunotherapy (OIT) is now recommended (4); it consists in a tolerance induction by daily ingestion of allergen. However, in the most severe cases which react with a very low quantity of cow's milk (CM), classic OIT cannot be initiated due to the impossibility of increasing the daily doses of CM. The adjunction of omalizumab, a monoclonal anti-IgE antibody, to OIT (oma-OIT), could be a safe and effective alternative in those cases; however, only a few studies have been published to decisively assess both the safety and efficiency of oma-OIT (5-8). The aim of our study was to evaluate both the effectiveness and safety in an oma-OIT protocol in children with persistent and very severe CMA.

In this study performed in Lyon, France, children who initiated an oma-OIT protocol for a persistent and very severe CMA were included. These patients were considered as severely allergic due to failure in initiation of a classic OIT to CM or an history of severe reaction with very small amounts of CM. Initiation of oma-OIT was decided in a concertation meeting of allergist experts in OIT. Briefly, the oma-OIT protocol was performed as following: (1) subcutaneous omalizumab was administrated at a frequency (every 2 or 4 weeks) and a dose that depended on the patient's weight and total IgE (same dose used as the one recommended for severe asthma); (2) after 16 weeks of omalizumab use alone, OIT was initiated. The induction phase began with the introduction of diluted amounts of CM (first daily dose: $1 \mathrm{mg}$ of CM proteins), followed by introduction of non-diluted doses and daily increases of CM doses until a predefined threshold. Oral food challenges (OFCs) were performed every month or two months at hospital, under the supervision of a medical team trained for the management of high-risk OFCs, in order to establish the cumulative tolerated dose (CTD) and the new threshold to reach by up-dosing. After each OFC, the new threshold was reached at home in one week, and maintained for several weeks, until the next OFC. Allergy tests were usually performed at each OFC (skin prick-test [SPT], specific IgE [sIgE] to CM and CM components and specific IgG4 [sIgG4] to CM, Phadia 250 Thermofisher, Uppsala, Sweden). Additionally, the patients and their parents reported any allergic reactions which occurred at home in a notebook, if applicable. We retrospectively collected the general characteristics of each patient at the initiation of the protocol. We also collected the initial grade and the most severe grade of allergic reactions to CM, using the Ring and Meissmer classification (9), the results of OFCs, the allergy tests and allergic reactions and the use of epinephrine during OFCs and at home. In addition, we collected responses from a satisfaction survey which was completed by the children and their parents after $\mathrm{OFC}_{4}$. 
In order to evaluate the effectiveness of the initiation of oma-OIT protocol, the primary outcome was the change in the CTD of ingested fresh $\mathrm{CM}$ between $\mathrm{OFC}_{1}$ and $\mathrm{OFC}_{4}$. The secondary outcomes were the changes in allergy tests, the occurrence of allergic reactions during OFCs and at home, and the results of the satisfaction survey. Statistical tests were performed using GraphPad Prism version 8.02 for Windows, GraphPad Software, La Jolla California USA, www.graphpad.com.

Eight children (3 boys and 5 girls, median age of 14.5 years [12-16]) with very severe CMA who received an oma-OIT treatment were included in this study (Table 1). The median administrated dose of omalizumab was $487.5 \mathrm{mg}$ [300-600] every 2 or 4 weeks. The median time between $\mathrm{OFC}_{1}$ and $\mathrm{OFC}_{4}$ was 127 days [84-427] for a total number of 1,342 ingested doses at home. After four OFCs (Table I and Figure S1), the CTD of $\mathrm{CM}$ was significantly increased (median $\mathrm{CTD}_{1}=0.96 \mathrm{mg}[0.64 ; 15.04]$ and $\mathrm{CTD}_{4}=160 \mathrm{mg}[12.8 ; 480]$, 167 -fold change, $p=0.0078)$. The anti-CM sIgE moderately decreased $\left(\mathrm{sIgE}_{1}=476.0 \mathrm{kU} / \mathrm{L}[227.0 ; 2048.0]\right.$ and $\operatorname{sIgE}_{4}=460.0 \mathrm{kU} / \mathrm{L}[97.2 ; 1482.0]$, change $\left.-3.4 \%, p=0.0391\right)$ and ratio anti-CM sIgG4 $/ \mathrm{sIgE}$ increased $\left(\mathrm{sIgG} 4 / \mathrm{sIgE}_{1}=8.6[1.8 ; 16.2]\right.$ and $\mathrm{sIgG} 4 / \mathrm{sIgE}_{4}=17.5[3.3 ; 223.1]$, change $\left.+103.5 \%, p=0.0313\right)$. There were no significant changes in SPT wheal and anti-CM component sIgEs. During the 32 OFCs performed in the 8 children under oma-OIT, 8 allergic reactions occurred in 6 children: 4 were grade 1 and 4 grade 2 according to the Ring and Meissner classification. These children were treated with oral antihistamine; none needed an epinephrine administration. Among the 1,342 ingested doses at home, two doses induced allergic reactions in two children: one grade 2 and one grade 3. The severe reaction was treated by epinephrine at home. Of note, a reactogenic cofactor (physical activity) without adaptation of the protocol was found for this severe reaction. Finally, all the children and their parents were globally very satisfied with the oma-OIT protocol, even if they expressed relative anxiety due to the risk of adverse reactions during the OFCs and the dose intake (Table S1). Interestingly, omalizumab injections were not considered as a constraint by most of the children and their parents (Table S1).

Thus, in this cohort of children with very severe CMA, the adjunction of omalizumab to OIT was effective and globally well tolerated, during the first months of the protocol. The main limitations of our study are the absence of a control group (placebo or no treatment), the limited size of our cohort, and the duration of follow-up. These are preliminary results and they need to be confirmed. Moreover, our patients were more severe than those included in previous studies, in terms of initial CTD and sIgE (5-8). Thus, this preliminary study encourages continuation of further oma-OIT protocols in cohorts of patients with very severe CMA.

Table 1. Individual clinical characteristics at inclusion and allergy features at first and fourth oral food challenges of patients under omalizumab and oral immunotherapy for cow's milk allergy.

Figure 1. Study protocol illustration. After a period of inclusion, corresponding to IgE-mediated diagnosis and prognosis evaluation, omalizumab is administrated alone for 16 weeks. Oral immunotherapy with fresh cow's milk is then initiated by oral food challenge $\left(\mathrm{OFC}_{1}\right)$ in order to determine the first cumulative dose $\left(\mathrm{CTD}_{1}\right)$ and then the first threshold to reach by up-dosing. OFCs were regularly performed to increase the threshold. Study outcomes were assessed after $\mathrm{OFC}_{4}$.

Figure S1. Evolution of allergy features throughout protocol.Each dot and line correspond to one patient. OFC: oral food challenge.

Table S1. Responses to satisfaction survey completed by children and their parents.

\section{References}

1. Flom JD, Sicherer SH. Epidemiology of Cow's Milk Allergy. Nutrients [Internet]. 2019 May 10 [cited 2020 May 23];11(5). Available from: https://www.ncbi.nlm.nih.gov/pmc/articles/PMC6566637/

2. Mousan G, Kamat D. Cow's Milk Protein Allergy. Clin Pediatr (Phila). 2016 Oct;55(11):1054-63.

3. Skripak JM, Matsui EC, Mudd K, Wood RA. The natural history of IgE-mediated cow's milk allergy. J 
Allergy Clin Immunol. 2007 Nov;120(5):1172-7.

4. Pajno GB, Fernandez-Rivas M, Arasi S, Roberts G, Akdis CA, Alvaro-Lozano M, et al. EAACI Guidelines on allergen immunotherapy: IgE-mediated food allergy. Allergy. 2018 Apr;73(4):799-815.

5. Nadeau KC, Schneider LC, Hoyte L, Borras I, Umetsu DT. Rapid oral desensitization in combination with omalizumab therapy in patients with cow's milk allergy. J Allergy Clin Immunol. 2011 Jun;127(6):1622-4.

6. Takahashi M, Soejima K, Taniuchi S, Hatano Y, Yamanouchi S, Ishikawa H, et al. Oral immunotherapy combined with omalizumab for high-risk cow's milk allergy: a randomized controlled trial. Sci Rep [Internet]. 2017 Dec 12 [cited 2020 Mar 25];7. Available from: https://www.ncbi.nlm.nih.gov/pmc/articles/PMC5727171/

7. Takahashi M, Taniuchi S, Soejima K, Hatano Y, Yamanouchi S, Kaneko K. Successful desensitization in a boy with severe cow's milk allergy by a combination therapy using omalizumab and rush oral immunotherapy. Allergy Asthma Clin Immunol [Internet]. 2015 May 28 [cited 2020 Mar 25];11(1). Available from: https://www.ncbi.nlm.nih.gov/pmc/articles/PMC4461908/

8. Wood RA, Kim JS, Lindblad R, Nadeau K, Henning AK, Dawson P, et al. A Randomized Double-Blind Placebo-Controlled Study of Omalizumab Combined with Oral Immunotherapy for the Treatment of Cow's Milk Allergy. J Allergy Clin Immunol. 2016 Apr;137(4):1103-10.e1-11.

9. Ring J, Messmer K. Incidence and severity of anaphylactoid reactions to colloid volume substitutes. Lancet. 1977 Feb 26;1(8009):466-9.

\section{Hosted file}

table 1-PAI-omalizumab and CM allergy.docx available at https://authorea.com/users/334525/ articles/460481-the-induction-of-oral-immunotherapy-is-facilitated-by-adjunction-ofomalizumab-in-children-with-very-severe-cow-s-milk-allergy

\section{Hosted file}

figure 1-PAI-oma and CM allergy.pptx available at https://authorea.com/users/334525/articles/ 460481-the-induction-of-oral-immunotherapy-is-facilitated-by-adjunction-of-omalizumabin-children-with-very-severe-cow-s-milk-allergy 